\title{
Real-Time Detection of Intermediates in Rhodium Catalyzed Hydrogenation of Alkynes and Alkenes by Dissolution DNP
}

Boeg, Peter Andreas; Duus, Jens Øllgaard; Ardenkjær-Larsen, Jan Henrik; Karlsson, Magnus; Mossin, Susanne

\section{Published in:}

The Journal of Physical Chemistry Part C

Link to article, DOI:

10.1021/acs.jpcc.9b01376

Publication date:

2019

Document Version

Peer reviewed version

Link back to DTU Orbit

Citation (APA):

Boeg, P. A., Duus, J. Ø., Ardenkjær-Larsen, J. H., Karlsson, M., \& Mossin, S. (2019). Real-Time Detection of Intermediates in Rhodium Catalyzed Hydrogenation of Alkynes and Alkenes by Dissolution DNP. The Journal of Physical Chemistry Part C, 123(15), 9949-9956. https://doi.org/10.1021/acs.jpcc.9b01376

\section{General rights}

Copyright and moral rights for the publications made accessible in the public portal are retained by the authors and/or other copyright owners and it is a condition of accessing publications that users recognise and abide by the legal requirements associated with these rights.

- Users may download and print one copy of any publication from the public portal for the purpose of private study or research.

- You may not further distribute the material or use it for any profit-making activity or commercial gain

- You may freely distribute the URL identifying the publication in the public portal 
Subscriber access provided by DTU Library

\section{C: Surfaces, Interfaces, Porous Materials, and Catalysis}

\section{Real-Time Detection of Intermediates in Rhodium Catalyzed Hydrogenation of Alkynes and Alkenes by Dissolution DNP}

Peter Andreas Boeg, Jens $\varnothing$. Duus, Jan Henrik Ardenkjaer-Larsen, Magnus Karlsson, and Susanne Mossin

J. Phys. Chem. C, Just Accepted Manuscript • Publication Date (Web): 25 Mar 2019

Downloaded from http://pubs.acs.org on March 27, 2019

\section{Just Accepted}

"Just Accepted" manuscripts have been peer-reviewed and accepted for publication. They are posted online prior to technical editing, formatting for publication and author proofing. The American Chemical Society provides "Just Accepted" as a service to the research community to expedite the dissemination of scientific material as soon as possible after acceptance. "Just Accepted" manuscripts appear in full in PDF format accompanied by an HTML abstract. "Just Accepted" manuscripts have been fully peer reviewed, but should not be considered the official version of record. They are citable by the Digital Object Identifier (DOI®). "Just Accepted" is an optional service offered to authors. Therefore, the "Just Accepted" Web site may not include all articles that will be published in the journal. After a manuscript is technically edited and formatted, it will be removed from the "Just Accepted" Web site and published as an ASAP article. Note that technical editing may introduce minor changes to the manuscript text and/or graphics which could affect content, and all legal disclaimers and ethical guidelines that apply to the journal pertain. ACS cannot be held responsible for errors or consequences arising from the use of information contained in these "Just Accepted" manuscripts. 


\title{
Real-Time Detection of Intermediates in Rhodium
}

\section{Catalyzed Hydrogenation of Alkynes and Alkenes}

\section{by Dissolution DNP}

Peter Andreas Boeg', Jens Øllgaard Duus', Jan Henrik Ardenkjær-Larsen², Magnus

Karlsson ${ }^{2, *}$, Susanne Mossin ${ }^{1, *}$

${ }^{1}$ Department of Chemistry, Technical University of Denmark, 2800 Kgs. Lyngby,

Denmark; ${ }^{2}$ HYPERMAG, Department of Health Technology, Technical University of

Denmark, 2800 Kgs. Lyngby, Denmark.

\begin{abstract}
The hydrogenation of alkynes and alkenes using a Shrock-Osborn catalysts was followed in-situ with dissolution dynamic nuclear polarization (dDNP) NMR. Natural abundance and ${ }^{13} \mathrm{C}$ labeled dimethyl acetylenedicarboxylate was hyperpolarized prior to hydrogenation using (1,4-bis\{diphenylphosphino\}butane)(2,5-norbornadiene) rhodium(I)
\end{abstract}


perchlorate, $[\mathrm{Rh}(\mathrm{NBD})(\mathrm{DPPB})] \mathrm{ClO}_{4}$. The increased signal-to-noise ratio of dDNP compared to conventional ${ }^{13} \mathrm{C}$ NMR allowed real-time detection of substrate and products as well as the modeling of the hydrogenation kinetics. The build-up of an intermediate was observed during interruption in hydrogen flow, substantiating the current view of the reaction mechanism. Selective inversion of the carbonyl NMR signal of the substrate was applied to demonstrate unequivocally that the new peak appearing in the spectrum originates from a reaction intermediate. The scope of the dDNP method for following reaction dynamics in real time was further demonstrated by substrate competition experiments.

\section{Introduction}

The need for more energy efficient and chemically specific reactions drives a continuous interest in the improved measurement of chemical kinetics and mechanistic understanding. While simple mechanisms satisfying chemical intuition and knowledge are 
the norm in homogeneous catalysis, the full, detailed picture is rarely achieved outside of the combined effort of advanced mechanistic elucidation and kinetic investigation. ${ }^{1}$

For reactions catalyzed by molecular compounds, modifications to the active site of the catalyst is an important means to improve specificity and efficiency. Successful modifications are dependent on the understanding of the steric and electronic properties of the catalyst's active sites. Insights here can come from spectroscopic identification of transient intermediate species in the reaction but also from details of the reaction kinetics. Conventional techniques for investigating the kinetics and mechanism of chemical reactions include infrared spectroscopy, ultraviolet-visible spectroscopy, mass spectrometry, and conventional (thermal) nuclear magnetic resonance spectroscopy (NMR). NMR is a valuable tool due to the high spectral resolution, but the inherently low sensitivity makes it a poor choice for investigating kinetics or mechanism of fast reactions or in diluted systems.

The sensitivity of NMR is enhanced significantly using various hyperpolarization (HP) techniques. These techniques include dissolution dynamic nuclear polarization (dDNP²) NMR and para-hydrogen induced polarization (PHIP). ${ }^{3-5}$ The HP techniques rely on 
overcoming the unfavorable Boltzmann distribution of traditional NMR, where only a small

fraction of the nuclei (some $10 \mathrm{ppm}$ for ${ }^{13} \mathrm{C}$ ) contribute to the signal. Applying dDNP this

fraction can be enhanced by several orders of magnitude $\left(10^{4}-10^{5}\right) .^{2}$ The signal is

however decaying exponentially back to thermal equilibrium with the NMR $T_{1}$ time constant, limiting the achievable duration of hyperpolarization experiments. Depending on the magnetic environment of the nucleus in the molecule, $T_{1}$ of ${ }^{1} \mathrm{H}$ are in the range of seconds whereas $T_{1}$ of ${ }^{13} \mathrm{C}$ can reach up to a few minutes. ${ }^{6}$ The largest $T_{1}$ values are found for carbon atoms not directly bound to other nuclei with non-zero nuclear spins. For this reason carbonyl carbon atoms are especially useful.

The dDNP technique relies on producing a sample where the target molecule and an electron polarizing agent (EPA, a free radical) is dissolved in a glassing matrix and subjected to low temperatures $(1-1.5 \mathrm{~K})$ and high magnetic field $(3-7 \mathrm{~T})$ in a dedicated instrument called a polarizer. The thermal spin polarization of the EPA electron, which is close to unity at these conditions, is then transferred to all the magnetically active nuclei in the target molecule by means of microwave irradiation. ${ }^{7}$ After reaching equilibrium in the polarization transfer the sample is dissolved in hot solvent and ejected from the 
polarizer for use in the experiments. ${ }^{2}$ Due to the polarization transfer mechanism, the dDNP technique can be applied to a wide range of target molecules making it more versatile than the techniques involving para-hydrogen which requires the presence of an unsaturated bond (PHIP) or coordinating moiety (SABRE). However, depending on the type of experiment performed, the need for molecules with nuclei in long $T_{1}$ positions may limit the number of suitable target molecules. The dDNP technique has mostly been used for biochemical and biomedical investigations. The number of reports in the literature dealing with purely chemical systems are fewer but include investigations of the DielsAlder reaction and the mechanistic features of catalytic polymerization reactions. ${ }^{8-10}$ In this work we use dDNP for the investigation of catalytic hydrogenation, see Scheme 1 for the reactions under investigation. The aim of the study was to use the superior signal strength to search for reaction intermediates, investigate the reaction kinetics and assess the mechanism of the catalyzed hydrogenation reaction. For this we chose a model system consisting of a Schrock-Osborn type catalyst ${ }^{11}$ and alkyne and alkene substrates in a polar solvent, acetone. The hydrogenation of alkenes and alkynes using cationic rhodium catalysts is a classical organometallic reaction. The mechanism has been 
investigated with a range of methods and the consensus is that for cationic rhodium catalysts the reaction proceeds by 1) coordination of the substrate and then 2) oxidative addition of hydrogen in the rate-determining step. ${ }^{1,12-14}$ Migration of hydrides to the alkyne is followed by elimination of the alkene product. This suggestion has been corroborated in the literature by the observation of a strong kinetic isotope effect. ${ }^{15}$ Despite attempts using ${ }^{1} \mathrm{H}$ NMR and electrospray ionization mass spectrometry; ${ }^{14}$ to our knowledge, a transient intermediate during catalytic hydrogenation by a Schrock-Osborn catalyst has never been observed. Here we present evidence of such an intermediate.

In order to follow the reaction and extract kinetic parameters using dDNP enhanced ${ }^{13} \mathrm{C}$ NMR spectroscopy the substrate should contain at least one carbon atom with a $T_{1}$ long enough to allow collecting NMR spectra with signals from both the substrate and the product. Furthermore the chemical shift of the carbon atom with long $T_{1}$ should change enough during the reaction to allow discrimination between substrate and product. This is true for carbonyl carbons in the substrates dimethyl acetylenedicarboxylate (DMAD), dimethyl maleate (DMM), n-butylacrylate (nBA) and methyl-3-butenoate (M3B), see Scheme 1 with measured $T_{1}$ in the range $35-60 \mathrm{~s}$. The carbonyl groups serve as a 
spectroscopic handle for following the kinetics of the reaction by ${ }^{13} \mathrm{C}$ NMR spectroscopy.

They are close to the reaction site on the molecule and are likely to participate in the coordination of the substrate to the Rh center. ${ }^{13} \mathrm{C}$ NMR has sufficient resolution to allow peaks of substrate and product to be well separated in the spectrum. Enrichment with ${ }^{13} \mathrm{C}$ greatly enhances the signal intensity, but is not necessary in order to follow the reaction with dDNP and determine kinetic parameters. Nevertheless, in this work the substrate was isotopically labeled in selected experiments in order to have the maximum intensity available.

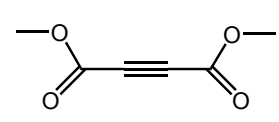

DMAD
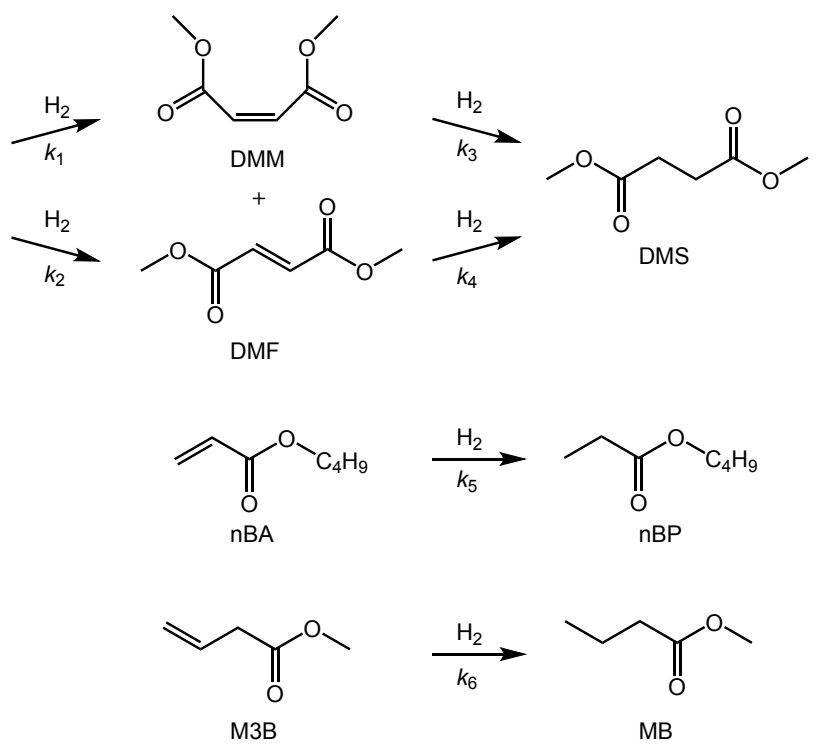

Scheme 1. Substrates and reactions under investigation. $k_{n}$ is the first order rate constant for the reactions given. 
The catalyst used is the cationic rhodium(I) complex of diphenylphosphinobutane (DPPB) obtained from hydrogenation of the norbornadiene-coordinated species (the precatalyst). The active catalyst is formulated as the 16 -electron square planar complex of rhodium(I) coordinated to DPPB and two solvent molecules (or one solvent molecule and the still-coordinated norbornene). The catalyst is selective for reaction with alkynes compared to alkenes, but will proceed to alkanes given enough time, see Scheme 1. Hydrogenation of dimethyl acetylenedicarboxylate (DMAD) proceeds to the alkene, which can be either the cis conformer, dimethyl maleate (DMM), or the trans conformer, dimethyl fumarate (DMF). Rhodium-catalyzed hydrogenation proceed through syn-addition giving the cis-conformer, DMM in large excess $\left(k_{1}>>k_{2}\right)$. Both DMM and DMF are hydrogenated to the same alkane, dimethyl succinate (DMS).

\section{Experimental Methods}

Dimethyl acetylenedicarboxylate (DMAD), dimethylmaleate (DMM), n-butylacrylate (nBA), methyl-3-butenoate (m3B), rhodium(III) chloride hydrate, $\mathrm{RhCl}_{3} \cdot \mathrm{xH}_{2} \mathrm{O}, 2,5$ norbornadiene, 1,4-bis(diphenylphosphino)butane (DPPB), acetone and the radical $\alpha, \gamma^{-}$ 
bisdiphenylene- $\beta$-phenylallyl (BDPA) were purchased from Sigma-Aldrich. $1{ }^{13} \mathrm{C}$ DMAD was obtained from Albeda Research ApS. 2,2',2"-(10-\{carboxymethyl\}-1,4,7,10tetraazacyclododecane-1,4,7-triyl)tris(3-\{benzyloxy\}propanoate)gadolinium(III) (Gadobop) was obtained from Bracco Imaging. Hydrogen gas (5.0) was purchased from AGA Denmark.

The synthesis of the catalyst precursor complexes was inspired by the work of Abel et. al. ${ }^{16}$ and Schrock et. al. ${ }^{17}$

Synthesis of $\left[\mathrm{Rh}_{2}(\mathrm{NBD})_{2} \mathrm{Cl}_{2}\right]$ (bis(2,5-norbornadiene)dichlorodirhodium): $1.05 \mathrm{~g}$ of $\mathrm{RhCl}_{3} \cdot \mathrm{H}_{2} \mathrm{O}$ was dissolved in $4 \mathrm{~mL}$ of ethanol. $4 \mathrm{~mL}$ of 2,5 -norbornadiene was added and the flask was sealed, heated and kept at $60{ }^{\circ} \mathrm{C}$ for 12 hours with stirring. The flask was subsequently cooled to room temperature and the precipitate was washed repeatedly with water and acetone and dried, resulting in a red, crystalline product. Yield: $2.20 \mathrm{~g}$ (95 $\%)$.

Synthesis of $\quad\left[\mathrm{Rh}(\mathrm{NBD})(\mathrm{DPPB}) \mathrm{ClO}_{4} \quad\right.$ (1,4-bis\{diphenylphosphino\}butane)(2,5norbornadiene) rhodium(I) perchlorate): $1.40 \mathrm{~g}$ sodium perchlorate was dissolved in 15 $\mathrm{mL}$ acetone, followed by the addition of $2.31 \mathrm{~g}\left[\mathrm{Rh}_{2}(\mathrm{NBD})_{2} \mathrm{Cl}_{2}\right] .4 .32 \mathrm{~g}$ of DPPB was slowly 
added with stirring. Stirring was continued for 30 minutes. The suspension was filtered and the precipitate was washed with dichloromethane. The volume of the filtrate was reduced to about $12 \mathrm{~mL}$ and ethanol was added in equal volume. Excess diethyl ether was slowly added, resulting in the precipitation of yellow crystals. The crystals were filtered off and washed with diethyl ether and air-dried. Yield: $3.11 \mathrm{~g}(96 \%)$. Purity $>99$ \% based upon ${ }^{31} \mathrm{P}$ NMR: $24.1 \mathrm{ppm}$ (doublet) with a ${ }^{1} \mathrm{~J}\left({ }^{103} \mathrm{Rh},{ }^{31} \mathrm{P}\right)$ coupling of $143.4 \mathrm{~Hz} .{ }^{18}$ Instrumentation:

Hyperpolarization was achieved using an Oxford Instruments HyperSense polarizer working at $3.35 \mathrm{~T}$ and $1.3 \mathrm{~K} .{ }^{13} \mathrm{C}$ NMR spectra were recorded on a Varian Inova $400 \mathrm{MHz}$ spectrometer equipped with a $10 \mathrm{~mm}$ broadband probe. Selected experiments were performed on the same spectrometer in a Shigemi tube using a $5 \mathrm{~mm}$ broadband probe. All time-resolved series of NMR spectra were recorded with a repetition time of $2 \mathrm{~s}$ between $20^{\circ}$ pulses. Most spectra were measured while hydrogen bubbled through the active volume of the NMR tube resulting in relative large width of the ${ }^{13} \mathrm{C}$ NMR peaks. Before extracting the integrals of the substrates and products the FID's were apodized 
with a $1 \mathrm{~Hz}$ exponential function and the spectra were baseline corrected with a "Whittaker smoother" polynomial fit.

Method:

For the experiments performed in this work, $40 \mu \mathrm{L}$ of a substrate sample (S) was hyperpolarized for $2 \mathrm{~h}$ (time constant of polarization build-up approx. $35 \mathrm{~min}$ ) and subsequently dissolved in $4 \mathrm{~mL}$ of acetone. $0.7 \mathrm{~mL}$ of this solution (PS) was then injected into a solution of the catalyst $(C)$ waiting in a $10 \mathrm{~mm}$ NMR tube. The catalyst solution had been prepared no more than 5 minutes before injection. To allow gas bubbling through the solution, the NMR tube was fitted with a thin glass tube ending in a low porosity (fine) glass frit below the active volume in the NMR tube. A hydrogen gas flow of $11.5 \pm 0.4 \mathrm{~mL}$ / min (0.5 mmol / min) was applied as described below, resulting in the gas being dispersed well in the solution as minuscule bubbles. The rapid convection caused by the gas was assumed to completely mix the samples between every pulse during timeresolved measurements.

Detailed description of experiments performed: 
Preparation of the substrate samples (S): For the experiments described in Figure 1-3, $250 \mu \mathrm{L}$ (approx. $289 \mathrm{mg}$ ) of DMAD was placed in an Eppendorf tube. $3.7 \mathrm{mg}$ BDPA radical and $6.2 \mathrm{mg}$ of a solution containing the gadolinium complex (100 $\mu \mathrm{mol}$ Gadobop / $\mathrm{g}$ DMSO) was added, yielding radical and gadolinium concentrations of $30 \mathrm{mM}$ and $2.5 \mathrm{mM}$ respectively. For the experiment in Figure 1 and 3 the DMAD was $100 \%$ isotopically labeled at one carbonyl position. For the experiment in Figure 2 natural abundance substrate was used. For the experiment described in Figure 4 three alkene esters (natural abundance); DMM (32 mg), nBA (28 mg) and M3B (22 mg) were mixed in an Eppendorf tube. This corresponds to equimolar amounts of each ester. BDPA and Gadobop were added to obtain the same concentrations as for the DMAD sample. The samples were polarized with microwave radiation at $93.99 \mathrm{GHz}$ at $100 \mathrm{~mW}$. The frequency was optimized and the optimum was found to be the same as for the standard trityl radical Ox063. After polarization of $\mathrm{S}$ the dissolution process takes place in $4 \mathrm{~mL}$ of acetone to obtain polarized solution PS. HyperSense works well with acetone as a solvent. The acetone is pressurized in an external vessel ( 4 bar $\mathrm{He})$ and heated until reaching 12 bars of pressure, which is set to trigger release. This happens at a lower temperature than for 
e.g. water due to the lower boiling point of acetone. Upon release the pressure of the solvent facilitates dissolution and extraction of the sample from the vessel in the polarizer.

The substrates are very soluble in acetone. The temperature after dissolution and after transfer through a non-heated transfer line was measured to be $30^{\circ} \mathrm{C}$ for selected experiments and assumed the same for remaining experiments. The temperature in the NMR spectrometer was set to the same temperature to minimize temperature gradients and thus all experiments reported here are performed at $30^{\circ} \mathrm{C}$. Blank experiments without catalyst consisting of injection of PS into $0.75 \mathrm{~mL}$ acetone and subsequent NMR measurement was performed several times in order to determine $T_{1}$ for DMAD, DMM, $\mathrm{nBA}$ and M3B independently.

Preparation of the catalyst solution C: A $10 \mathrm{mM}$ solution of $[\mathrm{Rh}(\mathrm{NBD})(\mathrm{DPPB})] \mathrm{ClO}_{4}$ in acetone was prepared. $0.75 \mathrm{~mL}$ of this solution was transferred to a $10 \mathrm{~mm}$ NMR tube. The tube was then fitted with the inset for hydrogen bubbling and prepared for injection. The concentration of catalyst and (combined) substrate after dissolution and injection is 
$3 \mathrm{mM}$ and $65 \mathrm{mM}$, respectively. There is at least $2 \%$ uncertainty on the concentrations due to the volatility of acetone combined with bubbling of hydrogen gas.

For the experiment in Figure 1 the tube is mounted in the NMR instrument and hydrogen bubbling was started at the moment of injection of PS into the catalyst solution. Acquisition of NMR spectra was initiated just after injection.

For the experiments in Figure 2 and 4 performed with the purpose of obtaining kinetic data; $30 \mathrm{~s}$ of hydrogen bubbling and $10 \mathrm{~s}$ of purging with nitrogen had taken place just before injection. Upon injection the hydrogen bubbling was restarted and acquisition of NMR spectra was initiated.

The experiment in Figure 3 (a) proceeded as for 2. Hydrogen bubbling was stopped about 12 seconds into the experiment to obtain the single spectrum shown.

For the experiment in Figure 3 (b) and (c), solution $\mathbf{C}$ was pretreated with hydrogen as in the experiment described for Figure 2 but with hydrogen bubbling for 5 min and no flushing with nitrogen. The solution was then transferred to a $5 \mathrm{~mm}$ Shigemi tube. Solution PS was injected and the solution left to react for approximately $30 \mathrm{~s}$. A $180^{\circ}$ pulse was 
applied at the frequency of the substrate peak with a width of $500 \mathrm{~Hz}(5 \mathrm{ppm})$ and then normal acquisition of NMR spectra every $2 \mathrm{~s}$ commenced.

\section{Results and discussion}

\section{Catalyst activation}

For the first experimental series in this work, we studied the hydrogenation reaction without activating the catalyst first. This allowed us to monitor the activation of the catalyst (the hydrogenation of norbornadiene) followed by the hydrogenation of the alkyne substrate, DMAD. We used isotopically labeled DMAD in order to have the maximum signal intensity in ${ }^{13} \mathrm{C}$ NMR. The time-resolved evolution in the integrated intensities of the individual peaks in the carbonyl region is given in Figure 1, left. The individual spectra are shown in Figure 1 right. We observe clearly the time-resolved development in the carbonyl peak of both substrate DMAD (152.0 ppm) and product DMM (165.6 ppm) and even the formation of DMS (172.5 ppm) from further reaction of DMM. An initiation period of around $37 \mathrm{~s}$ before DMM is formed correspond to activation of the catalyst. The formation of the alkane, DMS is observed prior to the full consumption of the alkyne, DMAD. In addition, it is clear that the product selectivity for the cis product, DMM is high. 
The formation of the trans product, DMF (164.9 ppm) is obscured in the experiment shown in Figure 1 due to the increased line width, but can be seen when hydrogen bubbling is terminated see Figure $3(a)$. The ratio of the areas under the NMR peaks estimated from the experiment shown in Figure 3 (a) is above 100:1 for DMM compared to DMF.
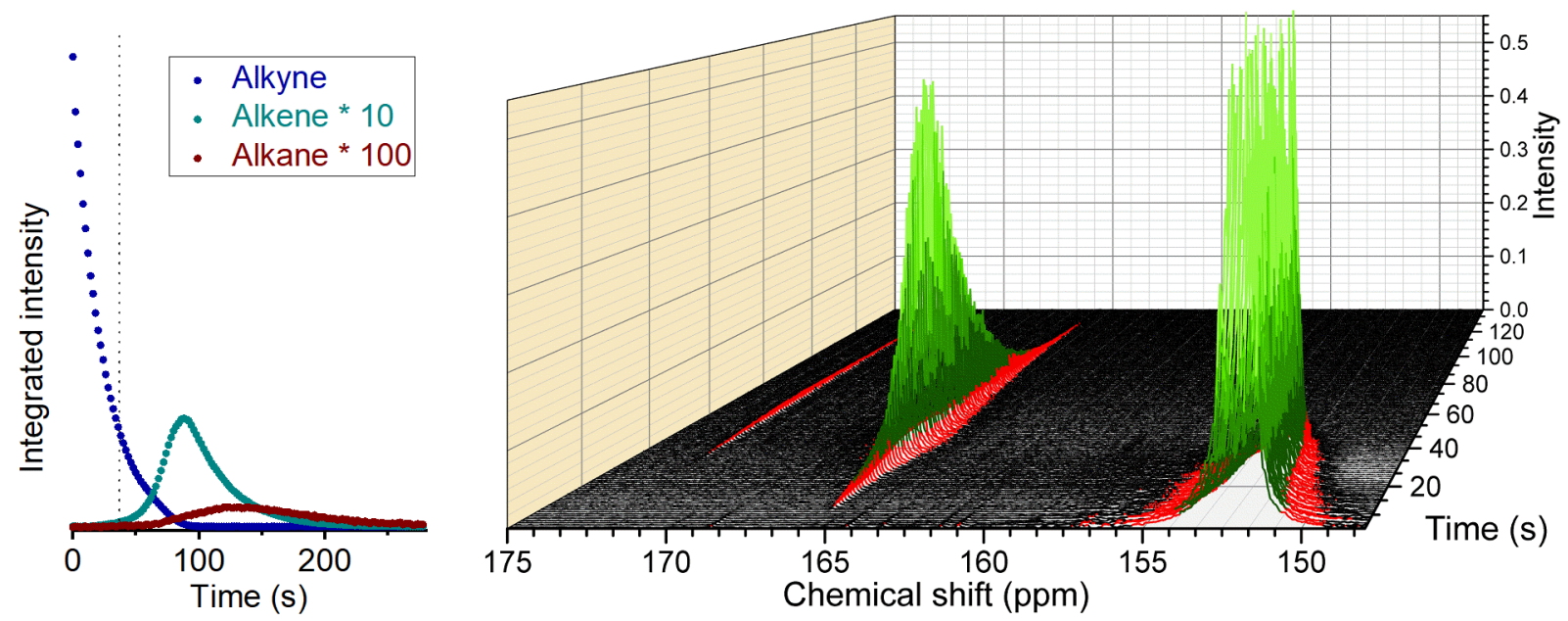

Figure 1. Time-resolved hyperpolarized ${ }^{13} \mathrm{C}$ NMR of the hydrogenation of DMAD with $[\mathrm{Rh}(\mathrm{NBD})(\mathrm{DPPB})] \mathrm{ClO}_{4}$. The signal decay is the combined result of the loss of polarization over time and the loss of signal due to the chemical reaction and the pulsing. Left: The change in integrated intensity of the carbonyl peaks of DMAD (152.0 ppm), DMM (165.5 ppm) and DMS (172.5 ppm) over time. An initiation period of $37 \mathrm{~s}$ corresponds to the 
activation of the catalyst. Right: The individual NMR spectra. The carbonyl peak of DMAD is cut off.

\section{Hydrogenation kinetics}

The kinetic modeling of the data given in Figure 1 was complicated by the in-situ activation of the catalyst. Simple models for the activation such as linear or exponential growth of active catalyst concentration did not lead to meaningful fits of the data. Actually, the activation of the catalyst may proceed in two steps since the protection group (norbornadiene) has two alkene groups coordinated to rhodium in the pre-catalyst, see Scheme 2 below. The partially hydrogenated protection group (norbornene) may still be coordinated to the rhodium center and this complex may also be an active catalyst, however with a different rate constant. We realized that we did not have sufficient information to select a reasonable model for the activation of the catalyst from these data and took a different approach towards reliable and reproducible kinetic data for the hydrogenation reaction. A new experiment was therefore designed: 
The catalyst was activated in the NMR tube by hydrogen bubbling prior to addition of the substrate. The hydrogen bubbling was stopped during injection at $t=0$ and initiated again immediately after. In this experiment natural abundance DMAD was used since signal-to-noise ratio was sufficient to follow the reaction from natural abundance substrate. In fact both the carbonyl carbon atom and the alkyne carbon atom in the substrate and the carbonyl carbon atom and the alkene carbon atom in the product have sufficiently long $T_{1}$ to follow the reaction for the purpose of obtaining rate constants, Figure 2. The methyl NMR ${ }^{13} \mathrm{C}$ signal from both substrate and product can also be clearly seen in the spectra and follows the same trend as the two other signal but artefacts from the decoupling of ${ }^{1} \mathrm{H}$ and the short $T_{1}$ of the order of $10 \mathrm{~s}$ precluded the use of the methyl signals for reliable fitting of the reaction kinetics.

The data of four replicates of the experiment was each fitted to a kinetic model implemented in SciPy ${ }^{19}$ assuming first order kinetics with respect to the substrate and zero order with respect to the hydrogen gas. The model consists of a first order rate law combined with loss of signal due to $T_{1}$ decay and due to pulsing. The reaction was monitored with ${ }^{13} \mathrm{C}$ NMR employing a nominal $20^{\circ}$ pulse angle. The pulse affects only the 
active volume of the sample (approximately $1 / 3$ of the total volume), but due to convection in the tube introduced by the bubbling of hydrogen, the observed signal loss corresponds to the application of a lower pulse angle. Hence, in the model the pulse angle is a fitted parameter for each individual experiment, see the Supporting Information. The fitted pulse angles are between 10 and $13^{\circ}$ for all experiments. The results of the fits are collected in Table 1 and simulations of the data using the fitted parameters are given as solid lines in Figure 2. The further conversion to DMS was not taken into account in the model since the signal from DMS was too modest using natural abundance substrate. $T_{1}$ of individual atom positions were investigated independently in three replicates by hyperpolarizing substrate with no catalyst present but with otherwise identical conditions. The $T_{1}$ decay was fitted using a simple exponential model. During fit of kinetic data, $T_{1}$ for the carbonyl position of DMM was fixed to the separately measured value of $60 \mathrm{~s}$. The other three measured $T_{1}$ values were used to set guess values and boundary conditions $( \pm 5 \mathrm{~s})$ for the parameters during fit of kinetic data. The results are given in Table 1. More details about the kinetic modeling are given in the Supporting Information. 
Table 1: Model parameters and $T_{1}$ of ${ }^{13} \mathrm{C}$ nuclei. $T_{1}$ values are given for the carbonyl $\mathrm{C}$ atoms unless another position is given.

\begin{tabular}{|c|c|c|c|}
\hline Substrat & $T_{1} / \mathrm{s}$ & $T_{1} / \mathrm{s}$ & $k / s^{-1}$ \\
\hline & $\begin{array}{l}\text { fitted from } \\
\text { experiment } \\
\text { without } \\
\text { catalyst }^{a}\end{array}$ & $\begin{array}{l}\text { fitted during } \\
\text { catalysis }\end{array}$ & $\begin{array}{l}\text { fitted during } \\
\text { catalysis }\end{array}$ \\
\hline DMAD & $36(2)$ & $\begin{array}{l}39(1)^{b} \\
33(1)^{b} \text { (alkyne) }\end{array}$ & \multirow{2}{*}{$k_{1}=0.0050(8)^{\mathrm{b}}$} \\
\hline DMM & $60(2)$ & $\begin{array}{l}60 \text { (fixed) } \\
8(1)^{\mathrm{b}} \text { (alkene) }\end{array}$ & \\
\hline DMM & $60(2)$ & 60 (fixed) & \multirow{2}{*}{$k_{3}=0.00040^{c}$} \\
\hline DMS & - & $53^{c}$ & \\
\hline nBA & $52.1(2)$ & $54^{c}$ & \multirow{2}{*}{$k_{5}=0.0039^{c}$} \\
\hline$n B P$ & - & $50^{c}$ & \\
\hline M3B & $44.2(2)$ & $42^{c}$ & \multirow{2}{*}{$k_{6}=0.00083^{c}$} \\
\hline MB & - & $47^{c}$ & \\
\hline
\end{tabular}

${ }^{a} T_{1}$ values are fitted from three replicates. ${ }^{b}$ Values obtained from fit of four replicates. One experiment is shown in Figure 2, the rest in Figure S2. cValue obtained from fit of data from the experiment in Figure 4. 


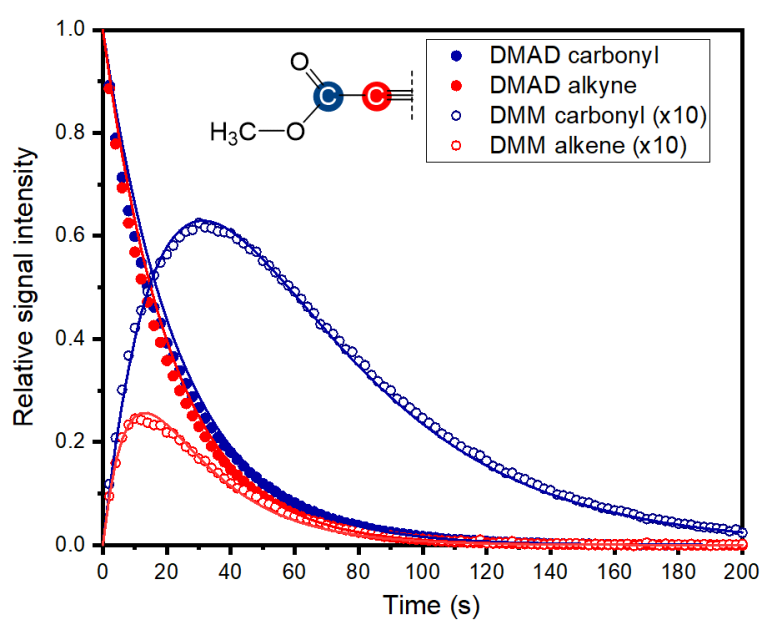

Figure 2. The change in ${ }^{13} \mathrm{C}$ NMR integrated signal intensity with time for 2 different carbon atoms during the conversion of DMAD to DMM with $[\mathrm{Rh}(\mathrm{NBD})(\mathrm{DPPB})] \mathrm{ClO}_{4}$. DMM signals are scaled $\times 10$ for easier comparison. The solid lines are simulated using the parameters obtained by modeling the time resolved development of intensity of two substrate peaks and two product peaks to first order reaction kinetics.

\section{Observation of reaction intermediates}

During the experiment with labeled DMAD we actively searched for reaction intermediates formed during the reaction since the superior peak intensity using dDNP 
on a labeled substrate should potentially allow such an observation. Three individual peaks in the carbonyl region were identified at 158.0, 162.4 and $176.7 \mathrm{ppm}$, see Figure S1. All three peaks were observable in the time interval $54-86 \mathrm{~s}$, but were completely absent both before and after. None of the three minor peaks are observable in the final thermal NMR spectrum. Consequently, the 17 individual spectra from Figure 1 obtained in this time interval were summed to give the spectrum shown as a blue line in Figure 3 (a). For the next experiment, isotopically labeled DMAD was hyperpolarized as before, but this time the hydrogen bubbling was terminated after $12 \mathrm{~s}$. The single-scan spectrum obtained just after interrupting the flow is shown as a black line in Figure 3 (a). Interestingly, the signal at $158.0 \mathrm{ppm}$ can now be clearly observed in a single spectrum. The 158.0 signal was repeatedly observed every time the $\mathrm{H}_{2}$ flow was turned off. The signals at 162.4 and $176.7 \mathrm{ppm}$ were not observed. An inversion experiment was performed in order to unequivocally determine, which peaks belong to species generated directly from DMAD. This experiment was originally devised by Zeng et al. ${ }^{10}$ The catalyst was preactivated in the normal NMR tube with the bubbling inset for $5 \mathrm{~min}$ and then transferred to a Shigemi NMR tube to minimize the volume of sample not being affected 
by pulsing. The hyperpolarized DMAD substrate (100\% labeled at one carbonyl position) was injected into the catalyst solution and once the turbulence had settled, a selective $180^{\circ}$ pulse was applied at the frequency corresponding to the DMAD carbonyl peak. Hence, the DMAD signal was inverted when measured just after the pulse, see Figure 3 (b). DMM and the intermediate (marked with green) are observed, whereas DMS and the species giving signals at 162.4 and 176.7 ppm (marked with yellow) are absent. The spectrum obtained $2 \mathrm{~s}$ later is given in Figure 3 (c). Here the signal corresponding to the $158.0 \mathrm{ppm}$ intermediate is also inverted. This is strong evidence that this intermediate originates directly from DMAD and is being formed continuously during the experiment.

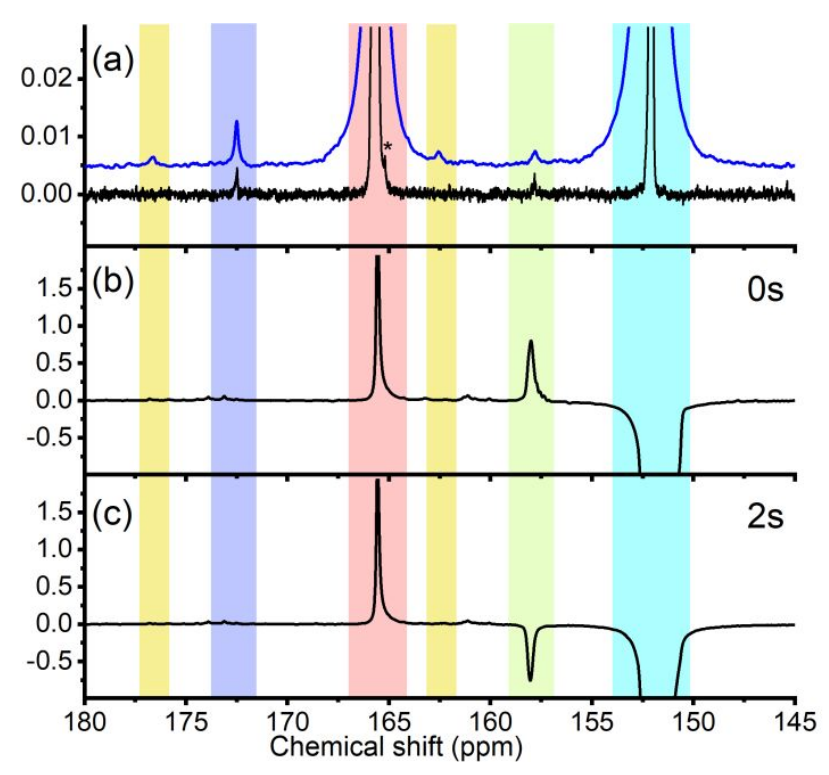


Figure 3. Zoom in of the carbonyl region of the ${ }^{13} \mathrm{C}$ NMR spectrum measured during the hydrogenation of DMAD with $[\mathrm{Rh}(\mathrm{NBD})(\mathrm{DPPB})] \mathrm{ClO}_{4}$. The position of the carbonyl signal of DMAD, DMM and DMS is marked with blue, red and purple, respectively. The signal position of DMF is marked with an asterisk. Peaks belonging to transient species are marked with green and yellow. (a) Blue line: Sum spectrum in the time interval 54-86 s of the spectra in Figure 1 obtained during hydrogen bubbling (the spectrum has been displaced along the y axis and rescaled for easy comparison). (a) Black line: Single spectrum collected shortly after stopping hydrogen bubbling in an analogous experiment.

(b) and (c): In a third experiment a pre-hydrogeneated catalyst was tranferred to a Shigemi tube and a $180^{\circ}$ pulse is applied at the frequency of the substrate peak. (b) show the spectra right after the flip pulse and (c) after additional $2 \mathrm{~s}$.

The currently accepted mechanism for the hydrogenation of alkenes by cationic rhodium catalysts with bidentate phosphine ligands is described by Jardine ${ }^{20}$. It is 
assumed to also be relevant for alkynes such as DMAD and is outlined in Scheme 2. The coordination of the substrate to $R h$ is suggested to be symmetric with the $C \equiv C$ axis perpendicular to the plane defined by rhodium and the 2 phosphorus atoms of the DPPB ligand. The final position in the plane is occupied by a solvent molecule indicated as $\mathrm{X}$. The reaction is suggested to proceed by oxidative addition of hydrogen to the 16-electron $\mathrm{Rh}$ (I) 2 to form 6 -coordinated 18 -electron $\mathrm{Rh}(\mathrm{III}) 3$. This is suggested to be the ratedetermining step. The migration of one hydride to the coordinated alkyne is suggested to result in alkenyl asymmetrically coordinated to $\mathrm{Rh}(\mathrm{III}) 4$. The migration of the second hydride results in a symmetrically coordinated alkene 5 . Rhodium is thus formally reduced to 16 -electron $\operatorname{Rh}(\mathrm{I})$. The alkene can either react further to obtain the alkane, be substituted with a solvent molecule or with a new alkyne for further reaction. 


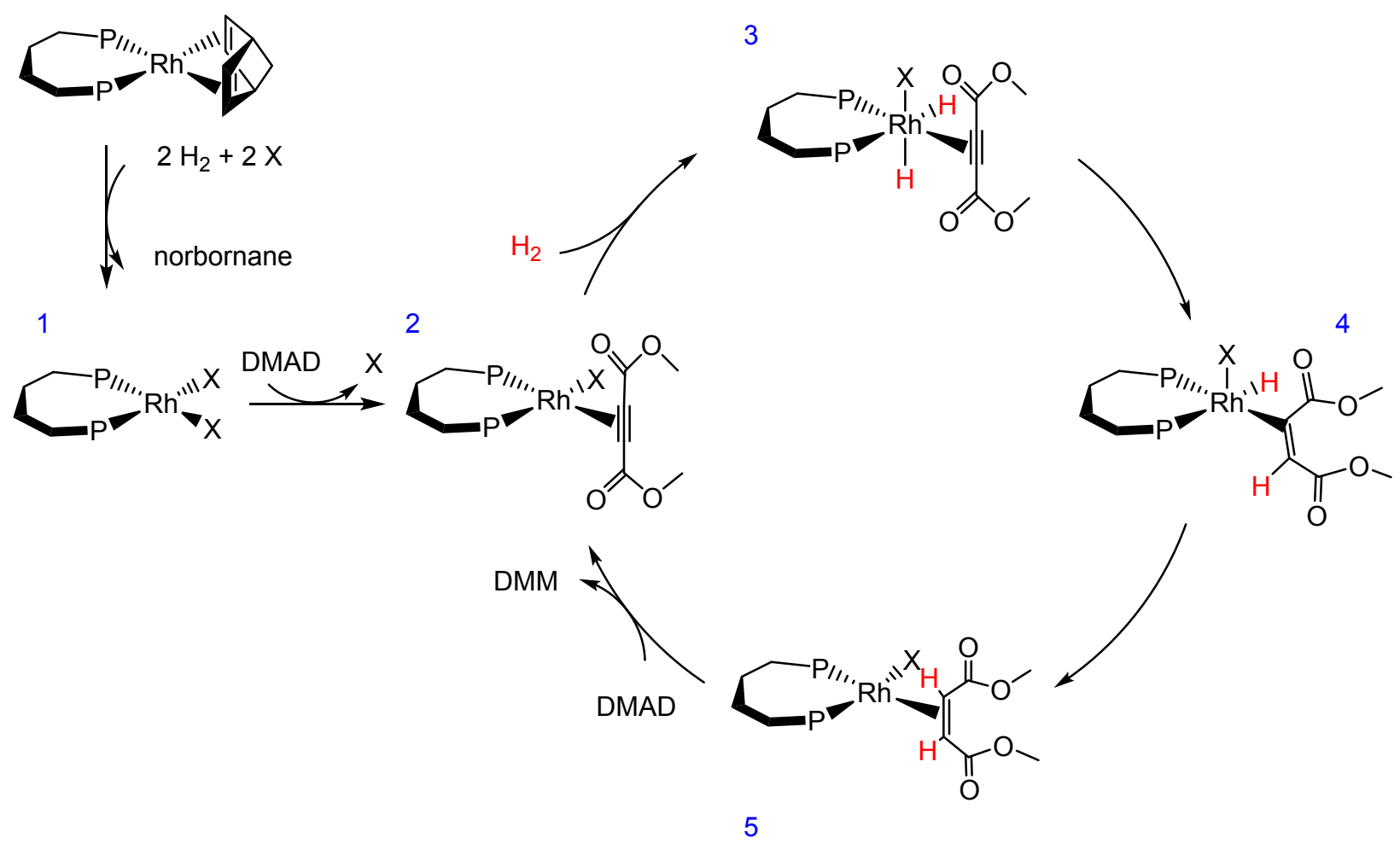

Scheme 2. Activation of the catalyst $[\mathrm{Rh}(\mathrm{DPPB})(\mathrm{NBD})]^{+}$and the suggested catalytic cycle of hydrogenation of DMAD to DMM. $X$ is a solvent molecule. Phenyl groups on phosphorous atoms of DPPB and the charge of +1 on all species are omitted for clarity.

Our results corroborate the suggestion above: Upon interruption of the hydrogen flow in a continuous experiment using DMAD, 2 is expected to accumulate and is the suggested assignment of the signal at $158 \mathrm{ppm}$. It is observable under reaction 
conditions, Figure 3 (a), blue line and Figure S1 although with a concentration so low that it is necessary to sum a number of spectra in order to observe it. The interaction with rhodium is expected to weaken the triple bond of DMAD due to $\pi$-backbonding from the electron-rich rhodium(I) to the antibonding $\pi^{*}$ orbital of the triple bond. We find the chemical shift of the carbonyl carbon of the observed intermediate to be close to the average of the chemical shifts of the carbonyl of the substrate alkyne and of the product alkene. The fact that only one signal can be assigned to the intermediate corroborates the symmetrical coordination. The substrate is only labelled with ${ }^{13} \mathrm{C}$ at one of the carbonyl positions and two signals are expected for any asymmetric species. Only a square planar $\mathrm{Rh}(\mathrm{I})$ coordinating the alkyne perpendicular to the plane would be completely symmetric.

A later intermediate such as the alkenyl intermediate 4 in Scheme 2, can be ruled out, since it is not symmetric with respect to the carbonyl carbon atoms. The observation of this intermediate corroborates that coordination of alkyne before oxidative addition of hydrogen is a viable reaction path.

For the other two observed transient peaks (at 162.4 and $176.7 \mathrm{ppm}$, marked yellow in Figure 3) there is less evidence to help the assignment. They are not observable in the 
experiment given in Figure 3 (b) and (c) despite the lower noise level. They are therefore likely to be later intermediates formed after the first hydrogenation step. Given that DMAD coordinated to the activated catalyst is observable, it is reasonable to search for a species corresponding to DMM coordinated to the activated catalyst (5 in Scheme 2). The carbonyl peak of this species would be expected to be found at a chemical shift intermediate between the carbonyl of DMM and DMS in analogy to the peak of coordinated DMAD suggested to be found at a chemical shift intermediate between DMAD and DMM. Nothing is observed here, so either the weaker association between rhodium and the alkene or the low concentration of DMM compared to DMAD has precluded observation of this species. Other suggested intermediates are the alkyne coordinated to the dihydrogen rhodium(III) species (3 in Scheme 2) and the alkenyl intermediate (4 in Scheme 2). The alkyne coordinated to the dihydride Rh(III) should be almost symmetric and give only one carbonyl peak (or two closely positioned carbonyl peaks) whereas the alkenyl intermediate (4 in Scheme 2$)$ is not symmetric and should result in two well-separated carbonyl peaks although one may be obscured if it is too close to the strong DMM peak. Exactly which effect the coordination to Rh would have on 
the chemical shifts of the carbonyl peaks of these intermediates is not straightforward to predict, and at the present time information is lacking in order to assign the observed peaks. A future assignment should be supported by e.g. a computational study or collection of other evidence not within the scope of this study.

\section{Competition experiments}

In order to demonstrate the potential of the dDNP method for substrate competition experiments equal molar amounts of three different alkene ester substrates (all natural abundance) were mixed then hyperpolarized and hydrogenated simultaneously. The substrates were dimethyl maleate, DMM, n-butylacrylate, nBA and methyl-3-butenoate, M3B, see Scheme 1. The alkenes were converted to the corresponding alkanes: Dimethyl succinate, DMS, n-butylpropionate, $\mathrm{nBP}$ and methylbutanoate, MB, respectively. The relative signal intensities of the ${ }^{13} \mathrm{C}$ NMR of the carbonyl are given as a function of time in Figure 4. As previously, the decay in signal intensity was modeled using a simple model with one rate constant for each competing reaction, allowing for $T_{1}$ decay for all species and decay due to pulsing. All constants determined for the conversion of all three 
substrates are given in Table 1. The simulated intensities using the fitted rate constants are given in Figure 4 as solid lines. Figure S3 show the simulated concentrations of substrate and product. The $T_{1}$ parameters of the carbonyl carbon are comparable for all three substrates in the range $44-60 \mathrm{~s}$. Figure 4 and $\mathrm{S} 3$ show that the conversion of nBA to $\mathrm{nBP}$ outcompetes the other two and indeed the fitted rate constant, $k_{5}$, for conversion of $\mathrm{nBA}$ to $\mathrm{nBP}$ is larger than $k_{3}$ with a factor of 10 and larger than $k_{6}$ with a factor of 5 . It is intuitive that the primary alkenes (nBA and $M 3 B$ ) are better substrates than the secondary alkene DMM, but perhaps surprising that $\mathrm{nBA}$ is significantly better than M3B. The difference between these two substrates is the number of connecting carbon atoms between the alkene and the ester group. The carbonyl oxygens in the ester group has been suggested to be able to coordinate to the rhodium center during the initial substrate binding. ${ }^{21}$ This clusters the coordination around rhodium that needs two open positions for oxidative addition of hydrogen in the rate-determining step. In addition, a more stable intermediate before the rate-determining step will slow the reaction down. For nBA the carbonyl oxygen is only connected through two carbon atoms to the alkene and cannot 
comfortably reach the second coordination site. For M3B three connecting carbons allow better reach.

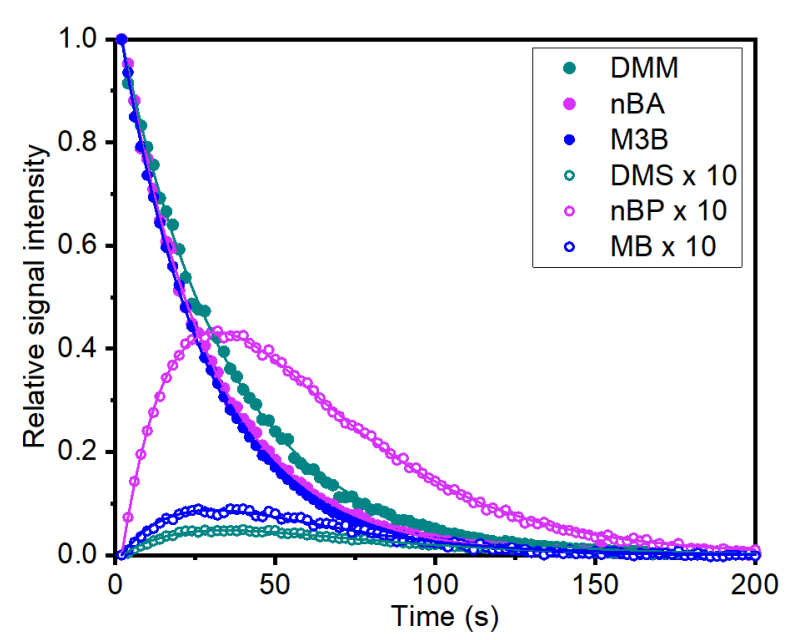

Figure 4. Open and closed symbols show the time-resolved development in the relative intensity of the ${ }^{13} \mathrm{C}$ NMR peaks in the carbonyl region of 3 different alkene esters during competitive hydrogenation using $[\mathrm{Rh}(\mathrm{NBD})(\mathrm{DPPB})] \mathrm{ClO}_{4}$ as the catalyst. The product peaks (open symbols) are scaled x10 for easier comparison. Lines show the simulated decay in the signal intensity modeled using first order kinetics analogously to the experiment in Figure 2. 
Alkynes are more reactive than alkenes towards hydrogenation and therefore it is not surprising that the first order rate constant $k_{1}$, for conversion of DMAD to DMM is significantly larger than $k_{3}$ for conversion of DMM to DMS. Comparing the results from the two kinetic experiments given above the ratio $k_{1} / k_{3}$ is found to be around 14 .

\section{Conclusion}

In this work we have demonstrated the application of dDNP to follow the hydrogenation of alkynes and alkenes by a cationic rhodium catalyst in solution. For kinetic studies natural abundance substrates can be used since the NMR relaxation parameters for all carbon atoms are adequate to follow the initial stages of the reaction. By using substrates with an isotopically labeled carbonyl carbon atom, the resolution and signal intensity are vastly enhanced and it is possible to observe transient intermediates during the hydrogenation in real-time. Stopping the hydrogen flow during the reaction resulted in the accumulation of a species which is identical to an observed intermediate in the hydrogenation reaction. This is suggested to be the substrate molecule coordinated to 
the activated rhodium catalyst. This corroborates the currently accepted mechanism where substrate binding is suggested to occur before oxidative addition of hydrogen to the rhodium complex. Inversion of the substrate NMR peak demonstrated unequivocally that the transient peak originates in a species formed directly from DMAD.

The dDNP protocol presented is also suitable for investigating competition between different substrates. This was demonstrated by following simultaneous hydrogenation of the alkene esters, $\mathrm{DMM}, \mathrm{nBA}$ and $\mathrm{M} 3 \mathrm{~B}$ with the same catalyst. It was shown that the catalyst had higher affinity for the less sterically hindered alkenes, $\mathrm{nBA}$ and $\mathrm{M} 3 \mathrm{~B}$, than for the more hindered alkene, DMM. It was also proven that $\mathrm{nBA}$ reacts faster than M3B by a factor of almost 5 . It was suggested to be due to the ester group in M3B being positioned for bidentate coordination. This allows stabilization of the reaction intermediate before oxidative addition of hydrogen (the rate-determining step) and thus leads to a slower reaction rate.

\section{ASSOCIATED CONTENT}


Details on the fitting of reaction kinetics and Figures S1, S2 and S3 are given in the

\title{
Supporting Information.
}

\author{
AUTHOR INFORMATION
}

Corresponding Authors: Magnus Karlsson, mkarls@dtu.dk; Susanne Mossin,

slmo@kemi.dtu.dk

Funding Sources

Danish National Research Foundation.

\author{
ACKNOWLEDGMENT
}

The Danish National Research Foundation is acknowledged for the grant DNRF124 for

HYPERMAG. PhD Sean Bowen is thanked for helpful discussions and help setting up

the inversion experiments. SM acknowledges the Lundbeck foundation for support.

\section{References}

(1) Sánchez-Delgado, R. A.; Rosales, M. Kinetic Studies as a Tool for the Elucidation 
of the Mechanisms of Metal Complex-Catalyzed Homogeneous Hydrogenation Reactions. Coord. Chem. Rev. 2000, 196 (1), 249-280. https://doi.org/10.1016/S0010-8545(99)00168-X.

(2) Ardenkjær-Larsen, J. H.; Fridlund, B.; Gram, A.; Hansson, G.; Hansson, L.; Lerche, M. H.; Servin, R.; Thaning, M.; Golman, K. Increase in Signal-to-Noise Ratio of $>10,000$ Times in Liquid-State NMR. Proc. Natl. Acad. Sci. 2003, 100(18), 1015810163. https://doi.org/10.1073/pnas. 1733835100.

(3) Bowers, C. R.; Weitekamp, D. P. Parahydrogen and Synthesis Allow Dramatically Enhanced Nuclear Alignment. J. Am. Chem. Soc. 1987, 109 (18), 5541-5542. https://doi.org/10.1021/ja00252a049.

(4) Glöggler, S.; Colell, J.; Appelt, S. Para-Hydrogen Perspectives in Hyperpolarized NMR. J. Magn. Reson. 2013, 235, 130-142. https://doi.org/10.1016/j.jmr.2013.07.010.

(5) Golman, K.; Axelsson, O.; Jóhannesson, H.; Månsson, S.; Olofsson, C.; Petersson, J. S. Parahydrogen-Induced Polarization in Imaging: Subsecond 13C Angiography. 
Magn. Reson. Med. 2001, 46, 1-5. https://doi.org/10.1002/mrm.1152.

(6) Bowen, S.; Hilty, C. Time-Resolved Dynamic Nuclear Polarization Enhanced NMR Spectroscopy. Angew. Chemie - Int. Ed. 2008, 47 (28), 5235-5237. https://doi.org/10.1002/anie.200801492.

(7) Wenckebach, T. Essentials of Dynamic Nuclear Polarization; Spindrift Publications, 2016.

(8) Lee, Y.; Heo, G. S.; Zeng, H.; Wooley, K. L.; Hilty, C. Detection of Living Anionic Species in Polymerization Reactions Using Hyperpolarized NMR. J. Am. Chem. Soc. 2013, 135(12), 4636-4639. https://doi.org/10.1021/ja4001008.

(9) Chen, C.-H.; Shih, W.-C.; Hilty, C. In Situ Determination of Tacticity, Deactivation, and Kinetics in [Rac-(C2H4(1-Indenyl)2)ZrMe][B(C6F5)4] and [Cp2ZrMe][B(C6F5)4]-Catalyzed Polymerization of 1-Hexene Using $13 \mathrm{C}$ Hyperpolarized NMR. J. Am. Chem. Soc. 2015, 137 (21), 6965-6971. https://doi.org/10.1021/jacs.5b04479. 
(10) Zeng, H.; Lee, Y.; Hilty, C. Quantitative Rate Determination by Dynamic Nuclear Polarization Enhanced NMR of a Diels-Alder Reaction. Anal. Chem. 2010, 82 (21), 8897-8902. https://doi.org/10.1021/ac101670n.

(11) Schrock, R. R.; Osborn, J. A. Catalytic Hydrogenation Using Cationic Rhodium Complexes. II. The Selective Hydrogenation of Alkynes to Cis Olefins. J. Am. Chem. Soc. 1976, 98 (8), 2143-2147. https://doi.org/10.1021/ja00424a021.

(12) Halpern, J. Mechanistic Aspects of Homogeneous Catalytic Hydrogenation and Related Processes. Inorg. Chim. Acta 1981, 50, 11-19. https://doi.org/10.1016/S0020-1693(00)83716-0.

(13) Kluwer, A. M.; Elsevier, C. J. Homogeneous Hydrogenation of Alkynes and Dienes. In The Handbook of Homogeneous Hydrogenation; 2008; pp 374-411. https://doi.org/10.1002/9783527619382.ch14.

(14) Luo, J.; Theron, R.; Sewell, L. J.; Hooper, T. N.; Weller, A. S.; Oliver, A. G.; Mclndoe, J. S. Rhodium-Catalyzed Selective Partial Hydrogenation of Alkynes. Organometallics $\quad 2015, \quad 34, \quad 3021-3028$. 
https://doi.org/10.1021/acs.organomet.5b00322.

(15) Jardine, F. H.; Osborn, J. A.; Wilkinson, G. Further Studies on the Homogeneous

Hydrogenation of Olefins Using Tris(Triphenylphosphine)Halogenorhodium(I)

$\begin{array}{lllllll}\text { Catalysts. } & \text { J. } & \text { Chem. } & \text { Soc. } & A & 1967, & 0\end{array}$

https://doi.org/10.1039/J19670001574.

(16) Abel, E. W.; Bennett, M. A.; Wilkinson, G. Norbornadiene-Metal Complexes and

Some Related Compounds. J. Chem. Soc. 1959, 3178-3182.

https://doi.org/10.1039/jr9590003178.

(17) Schrock, R. R.; Osborn, J. A. Preparation and Properties of Some Cationic Complexes of Rhodium(I) and Rhodium(III). J. Am. Chem. Soc. 1971, 93 (10), 2397-2407. https://doi.org/10.1021/ja00739a006.

(18) Slack, D. A.; Greveling, I.; Baird, M. C. 31P NMR Studies of Catalytic Systems Containing Rhodium Complexes of Chelating Chiral and Achiral Diphosphines. Inorg. Chem. 1979, 18(11), 3125-3132. https://doi.org/10.1021/ic50201a034. 
(19) Jones, E.; Oliphant, E.; Peterson, P. SciPy: Open Source Scientific Tools for Python. 2001.

(20) Jardine, F. H. Mechanism of Homogeneous Hydrogenation. In The Chemistry of the Metal-Carbon Bond, 2010; Vol. 4, pp 1049-1072. https://doi.org/10.1002/9780470771778.ch12.

(21) Meissner, A.; Alberico, E.; Drexler, H.-J.; Baumann, W.; Heller, D. Rhodium Diphosphine Complexes: A Case Study for Catalyst Activation and Deactivation. Catal. Sci. Technol. 2014, 4, 3409-3425. https://doi.org/10.1039/c4cy00497c. 


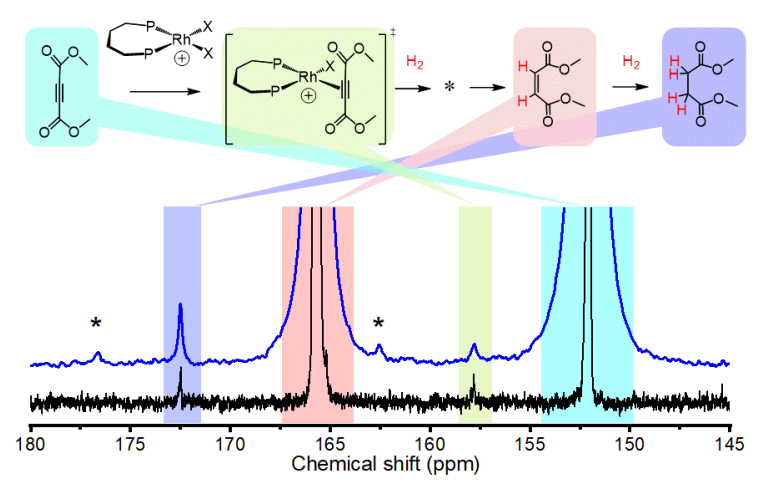

TOC Graphic

SYNOPSIS Dissolution Dynamic Nuclear Polarization NMR is used on ${ }^{13} \mathrm{C}$ marked substrates to observe transient species in real time in the hydrogenation of alkynes to alkenes using a Schrock-Osborn catalyst. Kinetic analysis and competition experiments have been performed using natural abundance substrates. 